\title{
Rapid On-Site Air Sampling with a Needle Extraction Device for Evaluating the Indoor Air Environment in School Facilities
}

\author{
Mitsuru Inoue,,$* *$ Ayako MizuguchI, ${ }^{* * *}$ Ikuo Ueta, ${ }^{* * *}$ Kazuya TAKAHASHI,* and \\ Yoshihiro SAITO* $*$ \\ *Department of Environmental and Life Sciences, Toyohashi University of Technology, Toyohashi 441-8580, \\ Japan \\ **Okazaki Technical Senior High School, Okazaki 444-8555, Japan \\ ***Department of Applied Chemistry, University of Yamanashi, Kofu 400-8511, Japan
}

\begin{abstract}
A rapid on-site air sampling technique was developed with a miniaturized needle-type sample preparation device for a systematic evaluation of the indoor air environments in school facilities. With the in-needle extraction device packed with a polymer particle of divinylbenzene and activated carbon particles, various types of volatile organic compounds (VOCs) were successfully extracted. For evaluating the indoor air qualities in school facilities, air samples in renovated rooms using organic solvent as a thinner of the paint were analyzed along with measurements of several VOCs in indoor air samples taken in newly built primary schools mainly using low-VOCs materials. After periodical renovation/maintenance, the time-variation profile of typical VOCs found in the school facilities has also been monitored. From the results, it could be observed that the VOCs in most of the rooms in these primary schools were at a quite low level; however, a relatively higher concentration of VOCs was found in some specially designed rooms, such as music rooms. In addition, some non-regulated compounds, including benzyl alcohol and branched alkanes, were detected in these primary schools. The results showed a good applicability of the needle device to indoor air analysis in schools, suggesting a wide range of future employment of the needle device, especially for indoor air analysis in other types of facilities and rooms including hospitals and hotels.
\end{abstract}

(Received February 7, 2013; Accepted March 7, 2013; Published May 10, 2013)

\section{Introduction}

Indoor air pollution, such as sick building syndrome (SBS) and multiple chemical sensitivity, have been recently focused on its effect on human health. ${ }^{1-3}$ In particular, children are most sensitive to these toxic chemical compounds, and therefore, precise determinations of volatile organic compounds (VOCs) in educational facilities could also be quite important. In 2002, the Ministry of Education, Culture, Sports, Science and Technology (MEXT) of Japan established a guideline for the concentration of formaldehyde and other VOCs, including toluene, xylene and $p$-dichlorobenzene, in the air environment of school facilities, based on reference values by the Ministry of Health, Labour and Welfare (MHLW) of Japan.,5 The regulation value of ethyl benzene and styrene were additionally listed in a guideline in 2004. To avoid any exposure to excessive chemical compounds, not only the detection of these regulated compounds, but also a sensitive determination of other VOCs might be necessary.

Gas chromatography (GC) is one of the most promising methods for the analysis of VOCs, and gas chromatographymass spectrometry (GC-MS) has been realized as a powerful tool for a sensitive determination of typical VOCs and semivolatile organic compounds (SVOCs) in air. ${ }^{6,7}$ For accurate and precise determinations of these VOCs, a sample preconcentration

† To whom correspondence should be addressed.

E-mail: saito@ens.tut.ac.jp process should still be performed before the analysis. Especially, the VOCs concentration related to school facilities could be at a quite low level; 8,9 therefore, an effective sample preparation process and subsequent separation/determination methods are needed. In terms of the sample preparation of VOCs in air samples, the use of a classic extraction cartridge, containing silica-gel or charcoal particles, is still accepted; however, these conventional methods require the use of a hazardous solvent or a special desorption device. A time-consuming analytical process with these sample preparation techniques could be another disadvantage, especially when a large number of sampling points must be necessary for a systematic indoor air analysis.

During past several years, we have developed novel needletype extraction devices, designed for the GC analysis of typical VOCs in air samples, ${ }^{10-13}$ including organic solvents often used in chemical laboratories, ${ }^{14}$ ethylene oxide,$^{15}$ aldehydes and ketones $^{16}$ and smoking-related compounds, ${ }^{17,18}$ along with the applications to real on-site sampling, such as fire investigation ${ }^{19}$ and breath analysis. ${ }^{20}$ These results clearly suggest a possibility of needle-type extraction to the analysis of indoor air samples, although the extraction medium packed in the needle should be further optimized to any particular analysis.

In the present work, double-bed-type sorbent consisting of divinylbenzene (DVB) and activated carbon (AC) particles ${ }^{21}$ was introduced as the extraction medium in the needle device for the analysis of indoor air in school facilities. Introducing the needle extraction device, VOCs in rooms of extensively renovated and 
newly-built primary schools were successfully determined. In addition, the time-variation profiles of typical VOCs found in these school facilities were also monitored.

\section{Experimental}

\section{Materials}

Toluene, ethylbenzene, xylene, styrene and $p$-dichlorobenzene ( $p$-DCB) were purchased from Tokyo Kasei Kogyo (Tokyo, Japan). The nitrogen gas ( $>99.99 \%$ purity) used for desorption of the extracted analytes and for the preparation of standard gas samples was purified by passage through a gas filter packed with molecular sieves $(5 \AA)$ to remove any undesirable organic contaminations. Smart Bag PA gas sampling bags were obtained from GL Sciences (Tokyo, Japan). A formaldehyde gas detector tube was purchased from Komyo Rikagaku Kogyo (Tokyo, Japan).

\section{Preparation of standard gas samples}

Standard VOC samples having desired concentrations were prepared as follows. First, an appropriate amount of the analyte was injected into a $1.0 \mathrm{~L}$-vacuum glass vessel and evaporated therein. Then, $1.0 \mathrm{~L}$ of pure $\mathrm{N}_{2}$ gas was supplied to the glass vessel. Next, a few milliliters of the above-mentioned gas sample were injected into a gas sampling bag and diluted with pure $\mathrm{N}_{2}$. As a result, a standard gas sample of a few hundreds of $\mathrm{n} \mathrm{L} \mathrm{L}^{-1}$ was prepared in the gas sampling bag. In order to obtain standard samples having lower concentrations, a similar process was repeated once more.

\section{Extraction and desorption procedure of VOCs}

A double-bed-type of extraction medium consisting of a polymer particle of DVB (25 mm packed section) as the first sorbent and an AC particle of Shincarbon ST $(5 \mathrm{~mm}$ packed section) as the second sorbent were employed. ${ }^{21}$ In a previous study, this extraction needle showed successful extraction and desorption performances for typical VOCs in indoor air samples, where relatively low-volatile compounds were extracted on the DVB sorbent, and high-volatile compounds were extracted on the AC sorbent. Although these sorbents were not specially designed for the extraction of specific compounds based on such as molecular shape recognition, relatively less-volatile compounds could be well-trapped on the DVB, and highly volatile compounds could be successfully trapped on the AC. ${ }^{21}$ These sorbents particles having a diameter of between 150 and $180 \mu \mathrm{m}$ were packed into a section of a specially prepared needle, $0.5 \mathrm{~mm}$ i.d., $0.7 \mathrm{~mm}$ o.d., $85 \mathrm{~mm}$ length with a tip hole. For sampling of indoor air samples, the extraction needle was attached to a commercially available vacuum sampling device (Komyo Rikagaku Kogyo, Tokyo, Japan), unless otherwise specified. The typical sampling volume was $50 \mathrm{~mL}$, and it took about 3 min to complete the sampling with the vacuum device. Additionally, at some of the sampling points, parallel sampling with a commercially available gas sampling pump (GSP-300FT-2, Gastec, Kanagawa, Japan) was also made, where a gaseous sample was introduced into the extraction needle at a flow rate of $20 \mathrm{~mL} \mathrm{~min}-1$ for $30 \mathrm{~min}$. All indoor sampling was carried out as follows: first, the room air was well-ventilated for $30 \mathrm{~min}$, where all of the windows and doors were opened; next, all of the windows and doors were closed for $5 \mathrm{~h}$ before air sampling. All air samples were collected at a height of $80 \mathrm{~cm}$ from the floor. Sampling was done for at least 5 points in all of the rooms. All the quantitative results shown in this study were the corresponding mean value of the sampling.
Table 1 Reference values of VOCs in a school facility by MEXT

\begin{tabular}{lc}
\hline & Reference value/ng L-1 \\
\hline Formaldehyde & 100 \\
Toluene & 260 \\
Ethylbenzene & 3800 \\
$p$-Dichlorobenzene & 240 \\
Xylene & 870 \\
Styrene & 220 \\
\hline
\end{tabular}

After extraction, the extraction needle was attached to an injection syringe containing $0.5 \mathrm{~mL}$ of $\mathrm{N}_{2}$ gas. Then, the extraction needle was inserted to a heated GC injection port, and the analytes were injected by the $\mathrm{N}_{2}$ gas, without any heating time in the injector before injection. It has been confirmed in preliminary experiments that the pre-heating time causes insufficient desorption of the extracted analytes, because of a backflow of the thermally desorbed analytes from the DVB layer to AC layer. ${ }^{21}$ The extracted samples could be stored in the needle at room temperature for at least three days. Formaldehyde was detected by a detector tube, where $1000 \mathrm{~mL}$ air samples were collected with a gas-sampling pump.

\section{GC measurements}

A JEOL JMS-Q1000GC Mk-II GC-MS instrument (JEOL, Tokyo, Japan) with a split/splitless injection port and a Shimadzu GC-2010 gas chromatography-flame ionization detector (GC-FID) (Kyoto, Japan) were used, and all of the injections were made by a split mode with a ratio of 5:1. GC separation was performed on an HR-5 fused-silica capillary column of $30 \mathrm{~m} \times 0.25 \mathrm{~mm}$ i.d. having a film thickness of $0.25 \mu \mathrm{m}(\mathrm{J} \& \mathrm{~W}$ Scientific, Folsom, CA).

As the carrier gas, He was employed at a typical head pressure of $50 \mathrm{kPa}$; and injector was maintained at $250^{\circ} \mathrm{C}$. The GC-MS interface temperature and the ionization voltage were set at $250^{\circ} \mathrm{C}$ and $70 \mathrm{eV}$, respectively, with electron impact ionization. The FID temperature was set at $250^{\circ} \mathrm{C}$. The column temperature was started at $40^{\circ} \mathrm{C}$ for $2.0 \mathrm{~min}$, and then programmed to $140^{\circ} \mathrm{C}$ at a rate of $20^{\circ} \mathrm{C} \mathrm{min}-1$ for a GC-MS measurement, and started at $50^{\circ} \mathrm{C}(1.0 \mathrm{~min}$ hold $)$, and then programmed to $190^{\circ} \mathrm{C}$ at a rate of $20^{\circ} \mathrm{C} \mathrm{min}-1$ for a GC-FID measurement. The mass spectrometer was operated in a total ion monitoring mode (TIM) with a $\mathrm{m} / \mathrm{z}$ range of 45 to 250 .

\section{Results and Discussion}

\section{Determination of VOCs by a needle-type extraction device}

In our previous study, several extraction and desorption performances of DVB/AC packed extraction needle were confirmed along with a satisfactory sample storage performance at room temperature, ${ }^{21}$ where the sampling volume was $600 \mathrm{~mL}$ at a flow rate of $20 \mathrm{~mL} \mathrm{~min}{ }^{-1}$ using a sampling pump on the basis of the guideline stated by MHLW. ${ }^{5}$ In this study, a sampling volume of $50 \mathrm{~mL}$ with a vacuum sampler was investigated using a DVB/AC packed needle for rapid on-site air sampling. The limit of quantifications (LOQs) of typical VOCs were less than $1 \mathrm{ng} \mathrm{L}^{-1}$ with a sampling volume of $50 \mathrm{~mL}$. This result clearly indicates adequate sensitivities of the method, because of a satisfactory sensitivity for VOCs in school facilities, as tabulated in Table 1. In addition, there were no significant differences between these sample collection methods for the 

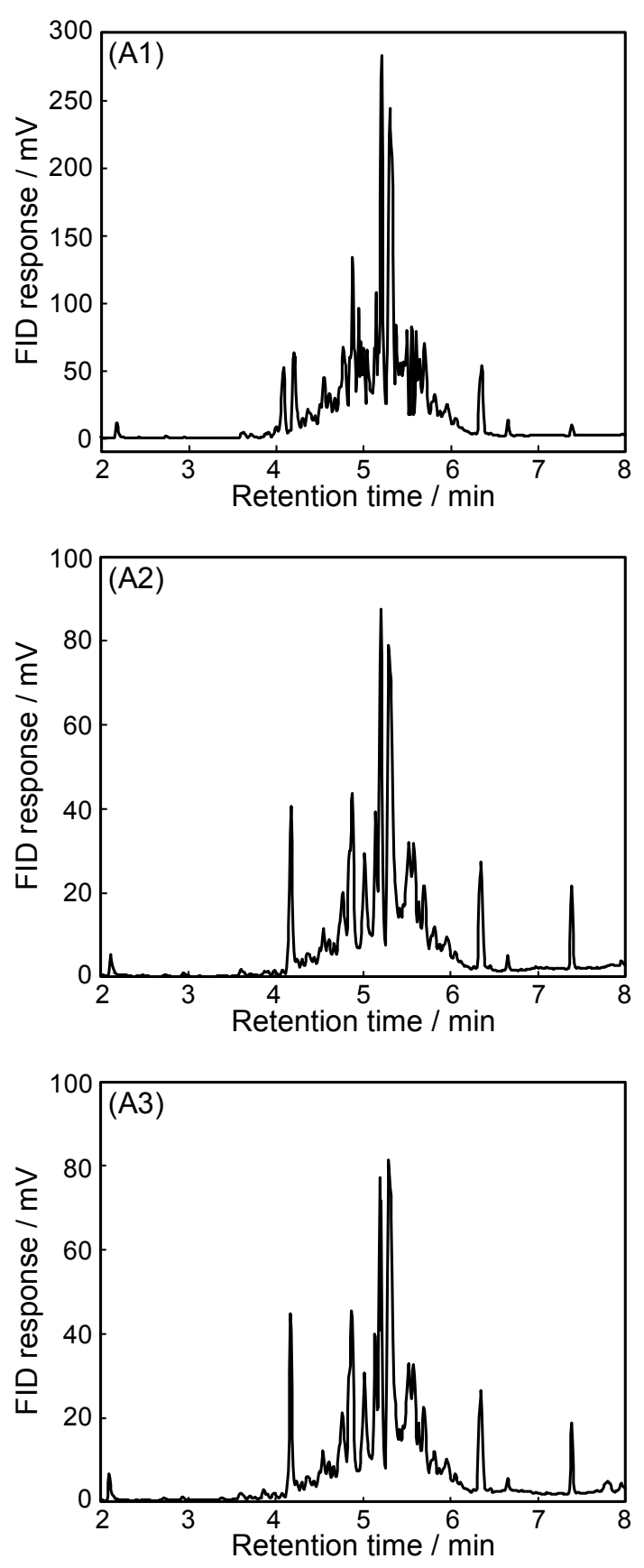

Fig. 1 Chromatograms for the separation of branched alkanes observed in three small teachers' rooms after a renovation in school A.

extraction performances, and the corresponding quantitative results in real indoor air samples. Therefore, the vacuum gas sampler was good enough to get satisfactory detectability for the VOCs in this work; however, to further improve the detectability, of course, the gas sampling pump could be introduced to increase the volume of the air sampling. The relative standard deviations (RSDs) of the peak areas were less than $5.0 \%(n=5)$ for real indoor air samples.

\section{VOCs in renovated rooms}

After a systematic optimization of the experimental conditions, indoor air samples taken from three small meeting rooms (in school A) were analyzed. The rooms $\left(26 \mathrm{~m}^{2}, 3.0 \mathrm{~m}\right.$ height $)$

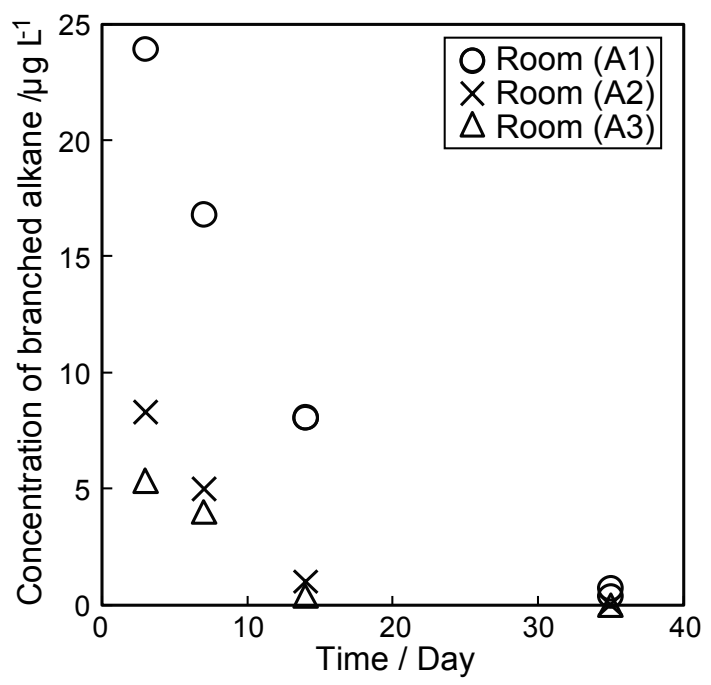

Fig. 2 Time profile of a branched alkane found in in-room air samples after a renovation in school A.

were renovated by replacing with new ceiling boards, and also by painting all of the walls. Figure 1 shows typical chromatograms for the separation of VOCs in air samples collected three days after completing the renovation. Significantly high concentrations of branched alkanes having carbon numbers of 10 and 11 were detected in all three rooms, probably because of using an organic solvent-based thinner. However, at this stage, these compounds were not yet regulated in the guidelines stated by MEXT and MHLW. Because of security reasons, all of the windows and doors in rooms B and $\mathrm{C}$ could be only opened during the day time, approximately from 9 am to $5 \mathrm{pm}$, while those in room A had to remain closed at all times. As can be expected, the level of VOCs in room A1 was significantly higher than those in rooms A2 and A3, although all of these chromatographic profiles are quite similar in Fig. 1. The time variations were monitored for up to 35 days after completing the renovation, as illustrated in Fig. 2. The amount of one of the branched alkanes (appeared at $5.2 \mathrm{~min}$ in Fig. 1) was plotted, where the amount was estimated as decane. From the plots, it is clearly found that the ventilation was quite effective to reduce the amount of VOCs; however, at the same time, more than several weeks should be necessary to reduce the level by about two orders of magnitudes, even with the ventilation normally carried out in this type of school facility renovation.

\section{VOCs in school rooms after a large renovation}

Indoor VOCs level after a large renovation in a primary school (school B) was studied. All 16 rooms listed in Table 2 were renovated as three groups, where each renovation was finished in June, September and December 2011. During these renovations, all of the ceiling boards, floor panel and wall paper were thoroughly replaced. As can be found in Table 2, the first sampling was made 3 days after completing the second renovation work in September, 2011. At that time, about 3 months had passed from completion of the first renovation in June, 2011, and third renovation was still in progress. The indoor air samples in third renovation area were collected 3 days after completion in December, 2011. Because these three sections were located in different buildings, no effect of 
Table 2 Concentration of VOCs in indoor air in a renovated primary school

\begin{tabular}{|c|c|c|c|c|c|}
\hline \multirow{2}{*}{ Room } & \multicolumn{5}{|c|}{ Concentration/ng L ${ }^{-1}$} \\
\hline & Toluene & Ethylbenzene & Xylene & Styrene & Benzyl alcohol \\
\hline Broadcasting room ${ }^{a}$ & 37.4 & 75.5 & 28.8 & 191 & 7.6 \\
\hline $\operatorname{Music} \operatorname{room}(\mathrm{B} 1)^{\mathrm{a}}$ & 117 & 73.1 & 32.6 & 159 & 82.1 \\
\hline Music room (B2) ${ }^{\mathrm{a}}$ & 125 & 45.2 & 22.8 & 65.2 & 87.6 \\
\hline Librarya $^{a}$ & 62.2 & $<1$ & $<1$ & 60.9 & $<1$ \\
\hline Computer room ${ }^{\mathrm{a}}$ & $<1$ & $<1$ & $<1$ & 27 & $<1$ \\
\hline Art and craft room ${ }^{\mathrm{a}}$ & 62.8 & 196 & 153 & 250 & 32.5 \\
\hline Science room ${ }^{\mathrm{a}}$ & 14.8 & 10.1 & $<1$ & $<1$ & N.D. \\
\hline Small gymnasium ${ }^{\mathrm{a}}$ & 41.7 & 26 & $<1$ & $<1$ & $<1$ \\
\hline Gymnasium $^{\mathrm{a}}$ & 9.3 & $<1$ & $<1$ & $<1$ & $<1$ \\
\hline Class room $(\mathrm{B} 1)^{\mathrm{b}}$ & 39.4 & 3.1 & $<1$ & 16.3 & 193 \\
\hline Class room $(\mathrm{B} 2)^{\mathrm{b}}$ & 60.6 & 39.1 & 16.6 & 127 & 259 \\
\hline Class room $(\mathrm{B} 3)^{\mathrm{b}}$ & 58.7 & 76.1 & 36.2 & 369 & 480 \\
\hline Class room (B4) ${ }^{\mathrm{c}}$ & 46.4 & 8.8 & 17.1 & $<1$ & 82.1 \\
\hline Class room (B5) ${ }^{\mathrm{c}}$ & 47.5 & 18.5 & 17.9 & $<1$ & 66.6 \\
\hline Class room $(\mathrm{B} 6)^{\mathrm{c}}$ & 32.7 & 17.6 & 17.1 & $<1$ & 62.4 \\
\hline
\end{tabular}

N.D.: Not detected.

a. Sampling was made after 3 months from the completion of the repair in September. b. Sampling was made after 3 days from the completion of the repair in September. c. Sampling was made after 3 days from the completion of the repair in December.
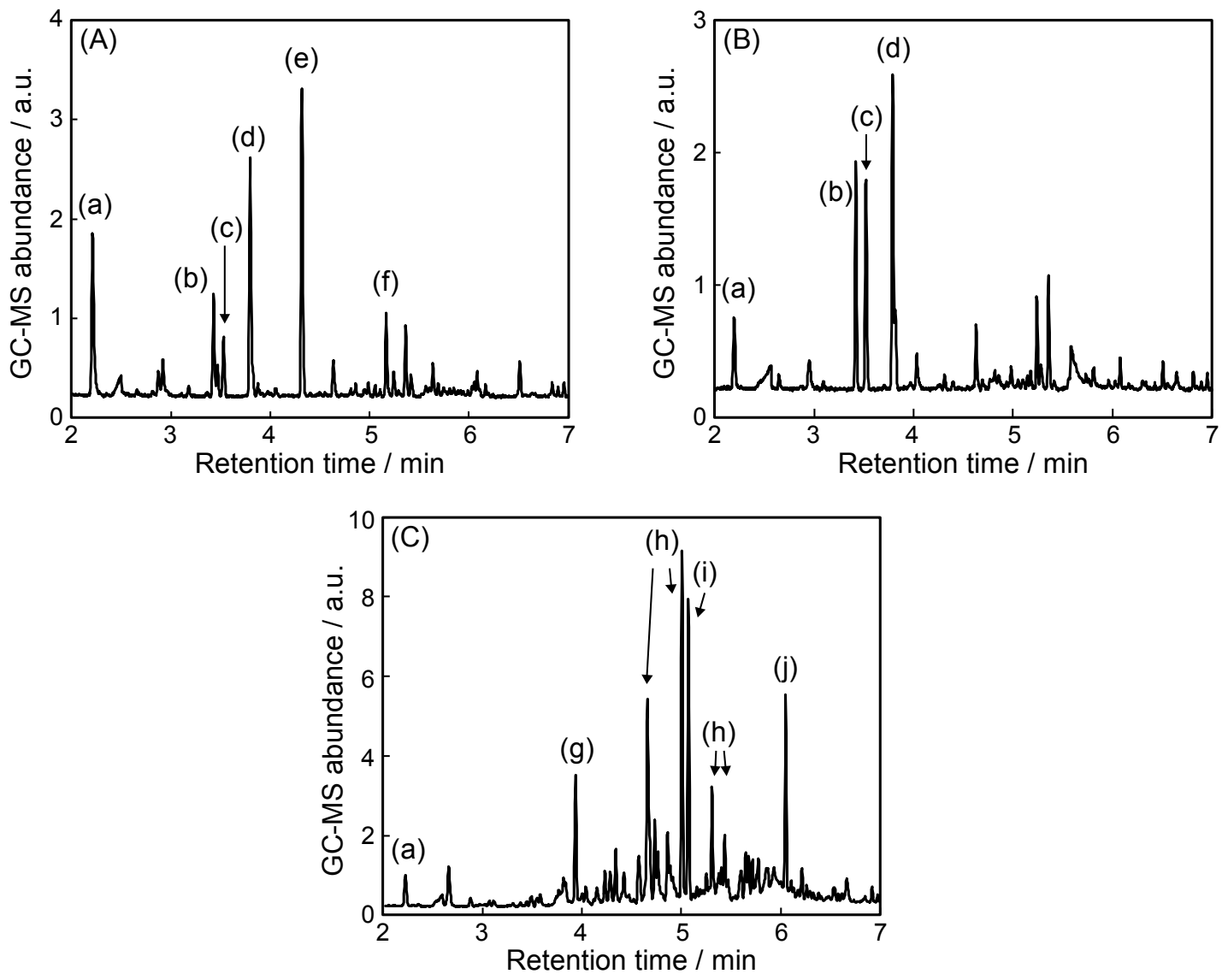

Fig. 3 Typical chromatograms for the separation of VOCs found in large-scale repaired school facilities (school B). (A) Music room, (B) art and craft room and (C) small gymnasium. Peaks: (a) toluene, (b) ethylbenzene, (c) $m$, p-xylene, (d) styrene, (e) $\alpha$-pinene, (f) 3-carene, (g) nonane, (h) alkylbenzenes, (i) decane, (j) undecane.

VOCs coming from other section was observed when monitoring outdoor air samples surrounding each building.

It is clearly found in Table 2 that the concentrations of all the regulated VOCs were lower than the corresponding reference values in all of the measured rooms in this school. The concentration of formaldehyde was less than $61 \mathrm{ng} \mathrm{L}^{-1}(50 \mathrm{ppm})$, and $p$-dichlorobenzene was not detected in all of the rooms. In the music rooms, relatively higher concentrations of VOCs were 
detected, and some terpene compounds were also detected, as can be found in Fig. 3(A). This is probably because these compounds were evaporated from specially designed soundproofing materials used for the walls and ceiling of the room. Relatively higher concentrations of VOCs were also observed in the art and craft room, as illustrated in Fig. 3(B). This could be associated with educational items, such as paints and adhesives. Some alkanes and alkyl benzenes were detected in a small gymnasium (Fig. 3(C)). These compounds might be generated from floor wax. The concentrations of these compounds were measured at three different height levels, i.e. at 2, 80 and $180 \mathrm{~cm}$ from the floor; however, no significant difference was found in all of the rooms measured. In addition, a relatively higher concentration of benzyl alcohol was detected in most of rooms, especially in class rooms B1 to B3. The low concentrations of benzyl alcohol in class rooms B4 to B6 could be attributed to a lower temperature of the sampling rooms.

Time-variation profiles of the concentrations of the VOCs in class room B3 were plotted in Fig. 4. As expected, all of the compounds decreased with the time; however, at the same time, it was confirmed that all of the concentrations of these VOCs reached a plateau, at about 2 months from completion of the repair, suggesting extensive air ventilation for at least several weeks should be necessary to minimize the exposure to these VOCs.

Long-term concentration profile in newly-built school facility

Because of recent increasing attention to the SBS, the use of paint and other miscellaneous materials with less VOCs emission have become popular. A school facility (school C) was built of the above-mentioned low VOCs materials. Table 3 summarizes the quantitative results of the VOCs in this newly-built school facility, where the air samples were collected in March, 2012. At the time of the sampling, about 7 months had already passed from completion of the construction. In this school facility, quite low concentrations of the regulated VOCs were observed, while $\alpha$-pinene and $d$-limonene (data not shown) were detected because of the employment of wood materials in interior materials. In this school, the library and computer room are connected by wood stairs, and this is probably because a high concentration of $\alpha$-pinene was detected in both rooms, as shown in Table 3. Decane and some branched alkanes having a carbon number of about 10 were only detected in the gymnasium (data not shown), and the total concentration of decane and these branched alkane in the gymnasium air sample is approximately $200 \mathrm{ng} \mathrm{L}^{-1}$ as decane. The source of these alkanes could also be attributed to the floor wax used in periodical maintenance in the gymnasium. Since all of the materials used in the renovation were carefully chosen not to have a high emission of formaldehyde, as expected, the concentration of formaldehyde

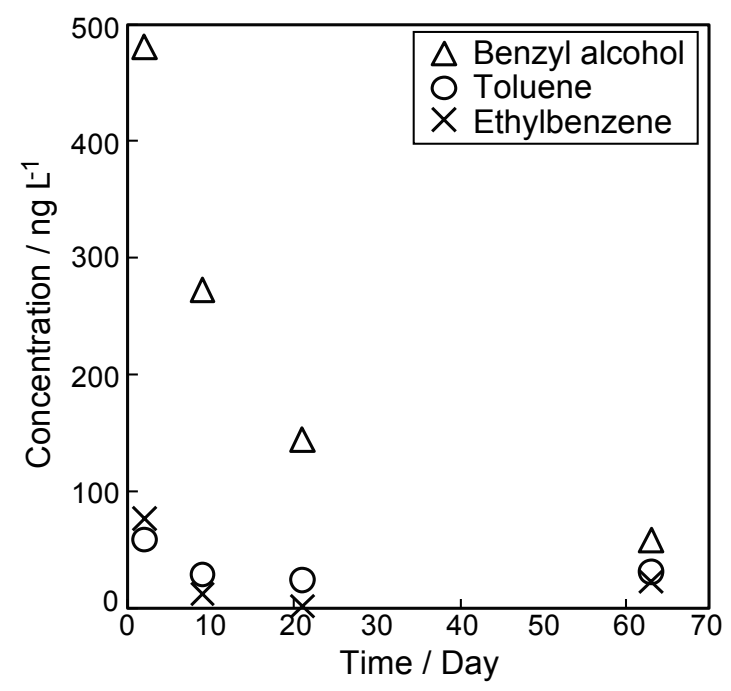

Fig. 4 Time variations of three VOCs after a large-scale repair in class room B3.

Table 3 VOCs found in a newly built primary school

\begin{tabular}{|c|c|c|c|c|c|}
\hline \multirow{2}{*}{ Room } & \multicolumn{5}{|c|}{ Concentration/ng L ${ }^{-1}$} \\
\hline & Toluene & Ethylbenzene & Xylene & Styrene & $\alpha$-Pinene \\
\hline Class room (C1) & 11.9 & 20.7 & 18.1 & 6.6 & 13.4 \\
\hline Class room (C2) & 14.8 & 7.5 & 11.9 & 12.6 & 21.8 \\
\hline Class room (C3) & 11.5 & 10.0 & 14.9 & 6.9 & 23.1 \\
\hline Class room (C4) & 8.5 & 14.2 & 14.2 & 8.5 & 31.9 \\
\hline Class room (C5) & 6.4 & 8.6 & 12.3 & 5.6 & 104 \\
\hline Class room (C6) & 6.9 & 10.8 & 11.2 & 15.7 & 87.3 \\
\hline Class room (C7) & 8.7 & 11.4 & 19.7 & 10.8 & 24.6 \\
\hline Class room $(\mathrm{C} 8)$ & $<1$ & 7.1 & 12.4 & 7.2 & 25.8 \\
\hline Student council room & 8.3 & 8.4 & 11.5 & 21.8 & 22.1 \\
\hline Infirmary room & 8.7 & $<1$ & 8.1 & 8.3 & N.D. \\
\hline Broadcasting room & $<1$ & $<1$ & $<1$ & 8.3 & N.D. \\
\hline Music room & 7.2 & $<1$ & 7.1 & $<1$ & 4.4 \\
\hline Music instrument room & 10.8 & 6.0 & 10.2 & 20.4 & 14.5 \\
\hline Library & 10.0 & 6.5 & 12.0 & 9.8 & 499 \\
\hline Computer room & 8.4 & 6.5 & 12.2 & 9.1 & 506 \\
\hline Art and craft room & 8.5 & 5.7 & 10.9 & 7.4 & 70.0 \\
\hline Home economics room & $<1$ & $<1$ & 12.1 & $<1$ & N.D. \\
\hline Printing room & 8.2 & 11.7 & 14.0 & 10.7 & N.D. \\
\hline Science room & 17.7 & 24.3 & 15.0 & $<1$ & 14.6 \\
\hline Small gymnasium & 9.6 & 9.6 & 11.7 & 16.8 & 15.9 \\
\hline Gymnasium & 2.2 & 2.6 & $<1$ & 10.0 & N.D. \\
\hline
\end{tabular}

N.D.: Not detected. 
Table 4 Long-term variation of the concentration of VOCs in a newly built school

\begin{tabular}{|c|c|c|c|c|c|c|}
\hline \multirow{2}{*}{ Room } & \multirow{2}{*}{ Measure period } & \multicolumn{5}{|c|}{ Concentration/ng L $\mathrm{L}^{-1}$} \\
\hline & & Toluene & Ethylbenzene & Xylene & Styrene & $\alpha$-Pinene \\
\hline \multirow[t]{2}{*}{ Class room (C6) } & March & 6.9 & 10.8 & 11.2 & 15.7 & 87.3 \\
\hline & August & 5.5 & $<1$ & 2.9 & 28.9 & 214 \\
\hline \multirow[t]{2}{*}{ Gymnasium } & March & 2.2 & 2.6 & $<1$ & 10.0 & N.D. \\
\hline & August & 3.9 & 4.3 & $<1$ & 12.1 & 85.4 \\
\hline \multirow[t]{2}{*}{ Music room } & March & 7.2 & $<1$ & 7.1 & $<1$ & 4.4 \\
\hline & August & 7.5 & 2.3 & $<1$ & 11.2 & 87.4 \\
\hline \multirow[t]{2}{*}{ Library } & March & 10.0 & 6.5 & 12.0 & 9.8 & 499 \\
\hline & August & 17.2 & 8.4 & 13.8 & 165 & 3253 \\
\hline \multirow[t]{2}{*}{ Computer room } & March & 8.4 & 6.5 & 12.2 & 9.1 & 506 \\
\hline & August & 16.7 & 8.1 & 13.9 & 168 & 3252 \\
\hline \multirow[t]{2}{*}{ Science room } & March & 17.7 & 24.3 & 15.0 & $<1$ & 14.6 \\
\hline & August & 13.5 & 7.6 & 20.0 & 48.4 & 73.8 \\
\hline
\end{tabular}

Average room temperature: March, $11.2 \pm 1.1^{\circ} \mathrm{C}$; August, $33.5 \pm 3.0^{\circ} \mathrm{C}$.

was less than $61 \mathrm{ng} \mathrm{L}^{-1}(50 \mathrm{ppm})$ in all the rooms.

Five months after the first sampling, air samples in this newly-built school facility were measured again (August 2012). Comparisons of the detected VOCs in March and August are shown in Table 4. When comparing the samples collected in March and August, the concentrations of most of the compounds were almost the same. However, the concentration of $\alpha$-pinene was significantly increased in the library and computer room. In addition, a higher level of styrene in these rooms was also found in August, 2012. This is probably due to evaporation during high temperatures in the summer, although a clear identification of the source of styrene is not established at this stage.

\section{Conclusions}

A needle-type extraction device was introduced to the GC-MS determination of VOCs in school facilities. The results showed that the needle extraction technique could be a suitable sample collection/extraction technique for indoor air analysis. The results also showed that the VOCs levels in most of the schoolrooms were thoroughly low level, although a relatively higher concentration of VOCs may be found in some room specially-designed for education purposes, and some non-regulated VOCs may also be found in school facilities. Taking advantage of the storage performance up to three days after the sampling, multipoint sampling at several rooms could be easily made. The developed technique will be a powerful tool for more systematic assessment of indoor air analysis, especially in newly build facilities and houses.

\section{Acknowledgements}

A part of this research was financially supported by Grants-inAid Scientific Research from the Japan Society for the Promotion of Science (JSPS). The authors acknowledge technical support from Prof. K. Jinno, Toyohashi University of Technology and Prof. S. Kawakubo, University of Yamanashi, and Dr. H. Wada, Shinwa Chemical Industries. One of the authors, M. I. also thanks to Mr. M. Morita and Mr. N. Hasegawa, Okazaki Technical Senior High School, and Mr. H. Osuga, Kira Senior High School for their helpful arrangements during this work.

\section{References}

1. P. A. Clausen, K. Wilkins, and P. Wolko, J. Chromatogr, A, 1998, $814,161$.

2. J. Jansz, in "Sick Building Syndrome", ed. S. A. AbdulWahab, 2011, Chap. 1, Springer-Verlag, Berlin Heidelberg.

3. S. N. Sinha, P. K. Kulkarni, N. M. Desai, S. H. Shah, G. M. Patel, M. M. Mansuri, D. J. Parikh, and H. N. Saiyed, J. Chromatogr., A, 2005, 1065, 315.

4. The Japan Ministry of Health, Labour and Welfare, Notice No. 261, 2003.

5. The Japan Ministry of Health, Labour and Welfare, Committee on Sick House Syndrome, Indoor Air Pollution Progress Report No. 1, 2000.

6. R. E. Clement and P. W. Yang, Anal. Chem., 2001, 73, 2761.

7. M. R. Ras, F. Borrull, and R. M. Marcé, Trends Anal. Chem., 2009, 28, 347.

8. G. Bertoni, C. Ciuchini, A. Pasini, and R. Tappa, J. Environ. Monit., 2002, 4, 903.

9. P. N. Pegas, C. A. Alves, M. G. Evtyugina, T. Nunes, M. Cerqueira, M. Franchi, C. A. Pio, S. M. Almeida, and M. C. Freitas, Environ. Geochem. Health, 2011, 33, 455.

10. M. Inoue, Y. Saito, I. Ueta, T. Miura, H. Ohkita, K. Fujimura, and K. Jinno, Anal. Sci., 2010, 26, 687.

11. I. Ueta and Y. Saito, Bunseki Kagaku, 2011, 60, 833.

12. Y. Saito, I. Ueta, M. Ogawa, A. Abe, K. Yogo, S. Shirai, and K. Jinno, Anal. Bioanal. Chem., 2009, 393, 861.

13. I. Ueta, K. Takahashi, and Y. Saito, Anal. Sci., 2012, 28, 953.

14. Y. Saito, I. Ueta, K. Kotera, M. Ogawa, H. Wada, and K. Jinno, J. Chromatogr., A, 2006, 1106, 190.

15. I. Ueta, Y. Saito, N. B. A. Ghani, M. Ogawa, K. Yogo, A. Abe, S. Shirai, and K. Jinno, J. Chromatogr., A, 2009, 1216, 2848. 
16. Y. Saito, I. Ueta, M. Ogawa, and K. Jinno, Anal. Bioanal. Chem., 2006, 386, 725.

17. Y. Saito, I, Ueta, M. Ogawa, M. Hayashida, and K. Jinno, $J$. Pharm. Biomed. Anal., 2007, 44, 1.

18. I. Ueta, Y. Saito, K. Teraoka, T. Miura, and K. Jinno, Anal. Sci., 2010, 26, 569.

19. I. Ueta, Y. Saito, K. Teraoka, H. Matsuura, K. Fujimura, and K. Jinno, Anal. Sci., 2010, 26, 1127.

20. I. Ueta, Y. Saito, M. Hosoe, M. Okamoto, H. Ohkita, S. Shirai, H. Tamura, and K. Jinno, J. Chromatogr., B, 2009, 877, 2551.

21. I. Ueta, A. Mizuguchi, K. Fujimura, S. Kawakubo, and Y. Saito, Anal. Chim. Acta, 2012, 746, 77. 